\title{
Endodontic Management of a Taurodontic Mandibular Second Molar
}

\author{
${ }^{1}$ Khaleel Ahamed Thaha, ${ }^{2}$ Nasil Sakkir, ${ }^{3}$ Mali G Nair, ${ }^{4}$ A Ajaz, ${ }^{5}$ Lija Issac Parackal
}

\begin{abstract}
Taurodontism is a dental anomaly in which the involved tooth has an enlarged and elongated pulp chamber with short roots. Endodontic treatment of a taurodont tooth presents challenges to a clinician for visibility, cleaning and shaping as well as obturation of the root canals. In this case report, a 16 -year-old female patient presented with spontaneous pain in relation to mandibular right second molar. On radiographic examination, the tooth had a carious lesion extending to the pulp chamber which was large and elongated with apically located furcation and two short roots. The taurodontic index (TI) for this particular tooth was 34.2, corresponding to mesotaurodontism. Endodontic treatment was successfully completed using lateral condensation technique and postendodontic restoration given.
\end{abstract}

Keywords: Bull teeth, Enlarged pulp chamber, Mesotaurodontism, Taurodontism.

How to cite this article: Thaha KA, Sakkir N, Nair MG, Ajaz A, Parackal LI. Endodontic Management of a Taurodontic Mandibular Second Molar. Cons Dent Endod J 2016;1 (1):14-17.

\section{Source of support: Nil}

Conflict of interest: None

\section{INTRODUCTION}

Taurodontism is a morphological developmental anomaly characterized by the lack of constriction at the level of the cementoenamel junction, vertically elongated pulp chambers, apical displacement of the pulpal floor and short roots. ${ }^{1}$ This anomaly is caused by the failure of Hertwig's epithelial sheath diaphragm to invaginate at the proper horizontal level. ${ }^{2}$ The term 'taurodontism' ('bull tooth') was derived from the Latin word 'tauros', which means 'bull' and the Greek word 'odus', which means 'tooth'. Sir Arthur Keith (1913) coined the term

\footnotetext{
${ }^{1}$ Senior Resident, ${ }^{2}$ Endodontist, ${ }^{3}$ Professor

4,5 Junior Resident

1,3-5Department of Conservative Dentistry and Endodontics Government Dental College, Thiruvananthapuram, Kerala India

${ }^{2}$ Department of Endodontics, Kamala Dental Speciality Hospital, Thiruvananthapuram, Kerala, India
}

Corresponding Author: Nasil Sakkir, Endodontist, Department of Endodontics, TC 2/453(22), Aisha Cottage, PT Chacko Nagar Medical College PO, Thiruvananthapuram, Kerala-695011 India, Phone: +91-9447895028, e-mail: nasil.sm@gmail.com 'taurodontism' to describe molar teeth resembling those of ungulates, particularly bulls. ${ }^{3}$ The prevalence of taurodontism ranges from 2.5 to $11.3 \%$. It can be unilateral or bilateral, with greater predilection for mandibular molars. Mandibular second molar is usually the most frequently involved tooth. ${ }^{4}$ Taurodontism usually occurs as an isolated anomaly, but it has also been associated with several developmental syndromes like Amelogenesis imperfecta, Down's syndrome, Hereditary ectodermal dysplasia, Klinefelter syndrome, Tricho-dento-osseous syndrome, Mohr syndrome, Wolf-Hirschhorn syndrome and Lowe syndrome. ${ }^{5}$

Shaw (1928) classified taurodontism as hypotaurodontism, mesotaurodontism and hypertaurodontism based on the relative displacement of the floor of the pulp chamber. ${ }^{6}$ In 1978, Shifman and Chanannel suggested a most widely accepted and used criteria. It was based on the distance from the lowest point of the roof of the pulp chamber to the highest point of pulp floor (V1), when divided by the distance from the lowest point of the roof of the pulp chamber to the root apex (V2) should be equal to or greater than $0.2 \mathrm{~mm}$. Also the distance from the highest point of pulpal floor to the cementoenamel junction (V3) should be greater than $2.5 \mathrm{~mm}^{7}$ (Fig. 1). Proper diagnosis and identification of the type of taurodontism is prudent to avoid procedural errors during endodontic therapy. This paper presents successful management of mesotaurodontism in mandibular right second molar tooth, along with its clinical considerations.

\section{CASE REPORT}

A 16-year-old female patient reported to the Department of Conservative Dentistry and Endodontics, Government Dental College, Thiruvananthapuram, with pain in lower right side of the jaw. Her medical history was noncontributory. On intraoral examination, there was a deep occlusal carious lesion on mandibular right second molar (tooth 47). Pulp vitality testing (Cold test and Electric pulp tesing) showed delayed response on 47. The tooth was sensitive to percussion. An intraoral periapical (IOPA) radiograph revealed the presence of a coronal radiolucency extending to the pulp chamber with elongated pulp chamber, apically displaced furcation and short roots (Fig. 2). The taurodontic index (TI) 
according to Shifman and Chanannel ${ }^{7}$ was 34.2. Based on the clinical and radiographic findings, a diagnosis of mesotaurodontism with symptomatic apical periodontitis was given to the mandibular right second molar tooth.

Inferior alveolar nerve block was administered to the patient using 2\% lidocaine with 1:100,000 epinephrine. Under rubber dam isolation, the access cavity preparation in relation to 47 was performed using an Endo Access bur and an Endo Z bur (Dentsply Tulsa). A huge pulp chamber was found, but the root furcation and the canal orifices were difficult to identify. Hence, a dental operating microscope (DOM; Seiler Revelation) was used to facilitate visualization and identification of the canals (Fig. 3). The three canals, mesiobuccal, mesiolingual and distal canals were located in the furcation area of pulpal floor. Pulp extirpation was performed, and the canals were thoroughly irrigated using $5 \mathrm{ml}$ of $3 \%$ sodium hypochlorite solution. An electronic apex locator (Root ZX, J Morita) was used to determine the working length, which was confirmed radiographically (Fig. 4). It was also identified that the mesiobuccal and mesiolingual

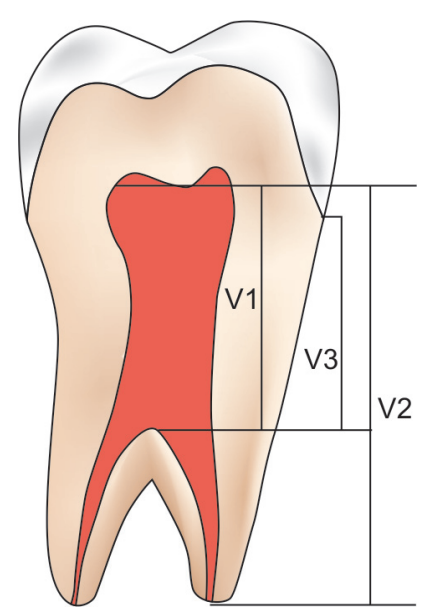

Fig. 1: Illustration for taurodontic index

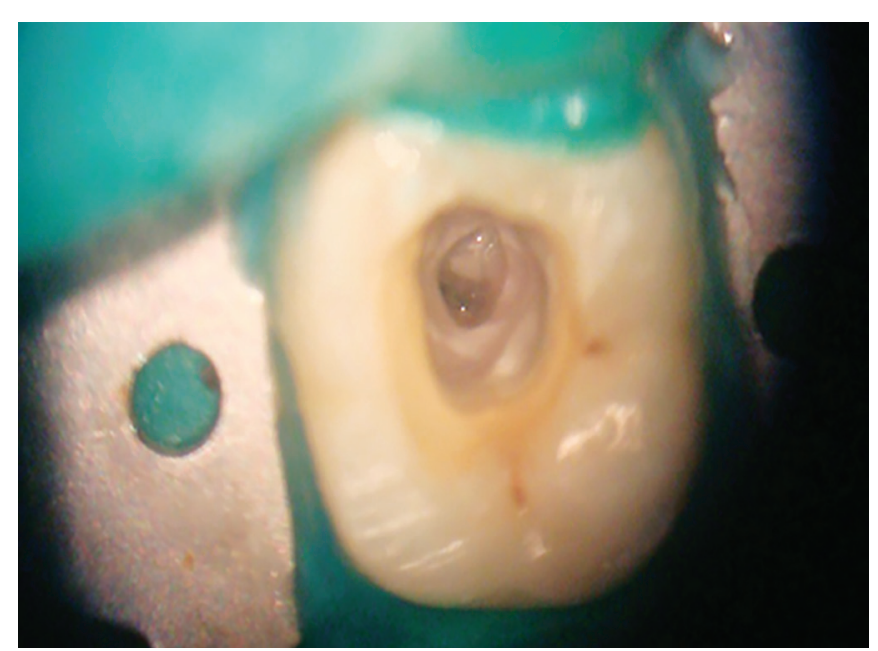

Fig. 3: Access cavity and pulp chamber under DOM (4x) canals had a Vertucci type II configuration, terminating at a single apical foramen. Cleaning and shaping of the root canals were performed using Hyflex (Coltene Whaledent) rotary files. The mesial and distal canals were instrumented up to $4 \% 30$ size. Final irrigation of the root canals was performed using 3\% sodium hypochlorite (manual dynamic agitation), 17\% aqueous solution of EDTA, and 2\% chlorhexidine gluconate solution.

A master cone radiograph was taken to confirm the fit of gutta-percha in all the canals (Fig. 5). The canals were dried using sterile paper points and obturated with gutta-percha cones and AH Plus sealer (Dentsply DeTrey) using a combination of lateral condensation and vertical compaction techniques. The gutta-percha cones were sealed at the level of canal orifices and a temporary restoration given (Fig. 6). The patient was recalled after 1 week and the postendodontic restoration was placed using Glass ionomer cement and composite restoration (Filtek Z350 XT, 3M ESPE). The patient was completely asymptomatic in the follow-up visits and the review radiograph taken after 1 year did not show any signs of

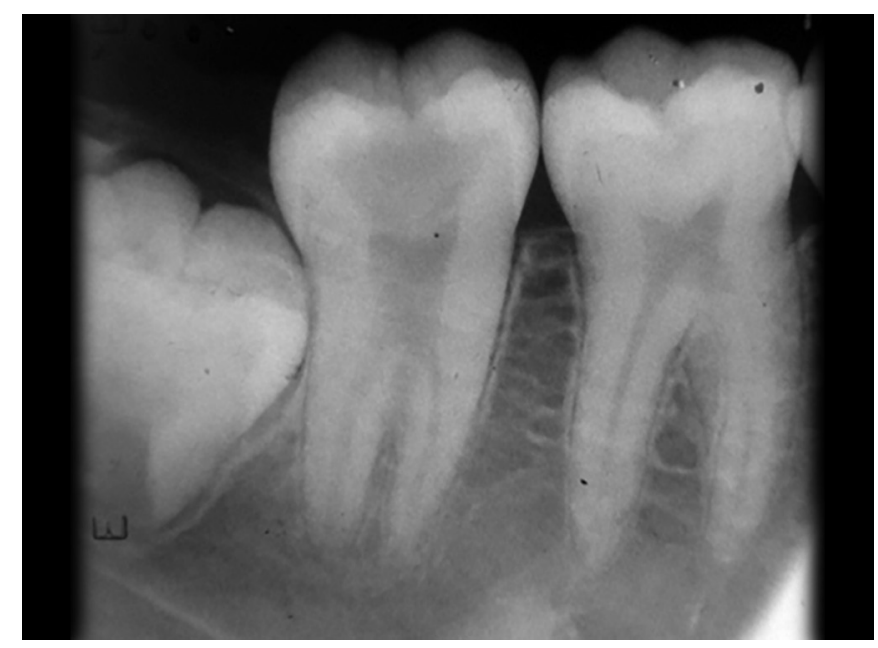

Fig. 2: Preoperative radiograph

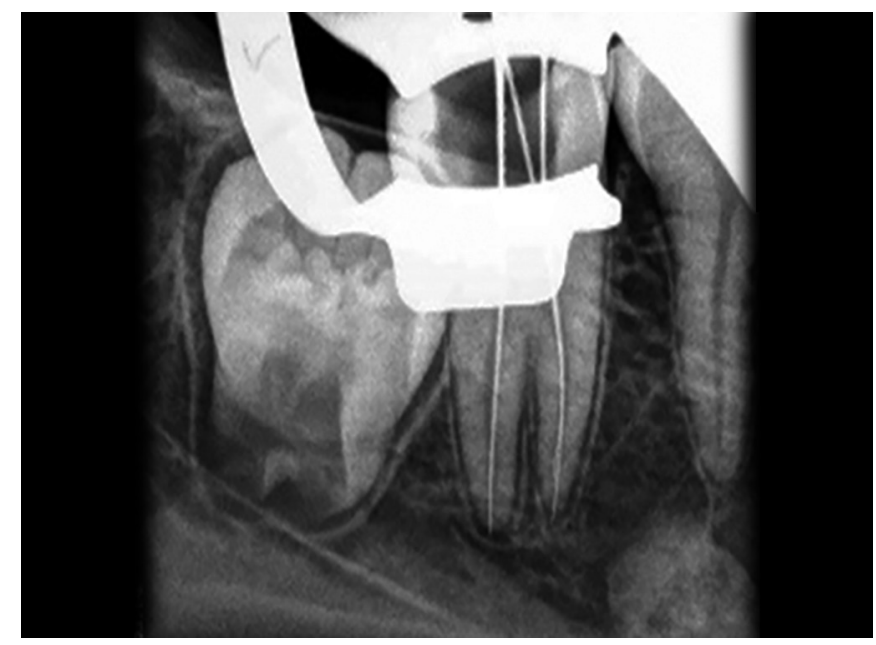

Fig. 4: Working length radiograph 


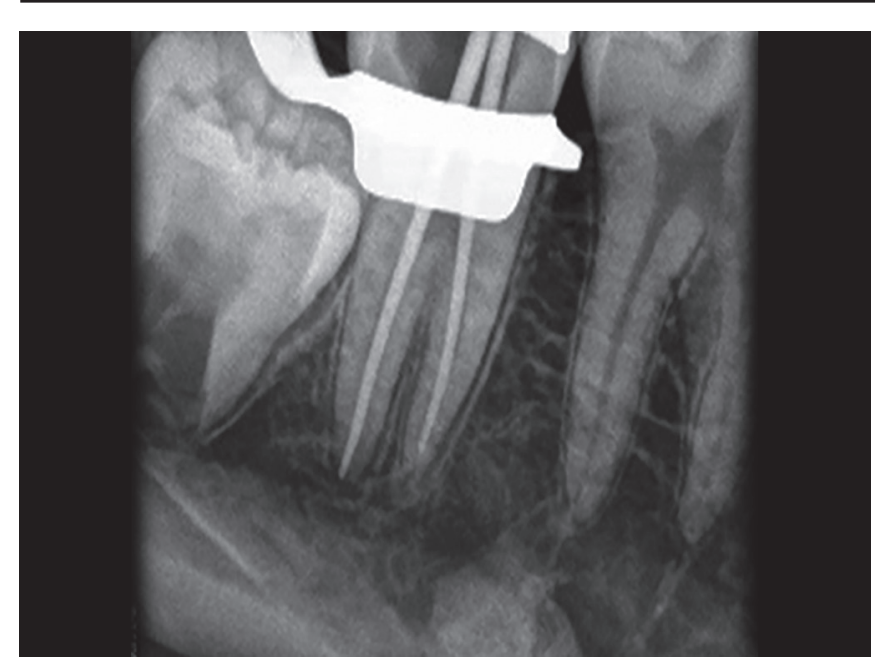

Fig. 5: Master cone radiograph

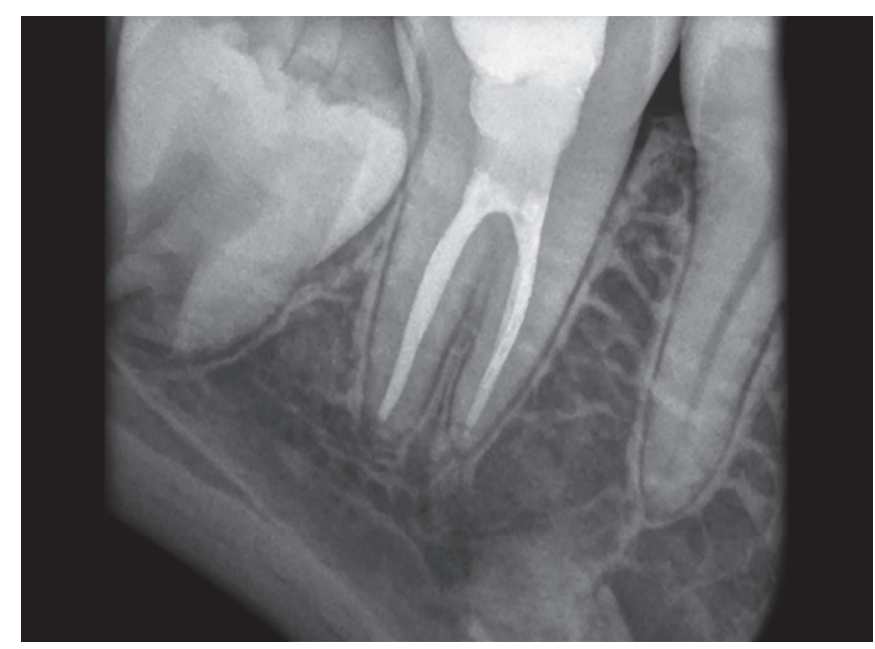

Fig. 7: Follow-up radiograph after 1 year

periapical lesion (Fig. 7). Patient was advised to report back for the placement of a full-coverage restoration after complete eruption of the mandibular third molars.

\section{DISCUSSION}

Endodontic treatment of a taurodontic tooth is a challenge as it exhibits variation in the size of the pulp chamber, apically positioned root canal orifices with short roots. Clinically, the taurodont crown has normal structure, form, color and texture; so taurodontism can only be diagnosed by radiographic examination. ${ }^{8}$ Shifman and Chanannel proposed an index to calculate the degree of taurodontism. According to this index, taurodontism is present if the distance from the lowest point of the roof of the pulp chamber to the highest point of pulp floor (V1) divided by the distance from lowest point of the roof of the pulp chamber to the root apex (V2) and multiplied by 100 is 20 or above. Taurodontic index is 20 to 30 for hypotaurodontism, TI 30 to 40 for mesotaurodontism and TI 40 to 75 for hypertaurodontism. ${ }^{7}$ According to Feichtinger and Rossiwall, the distance from the bifurcation

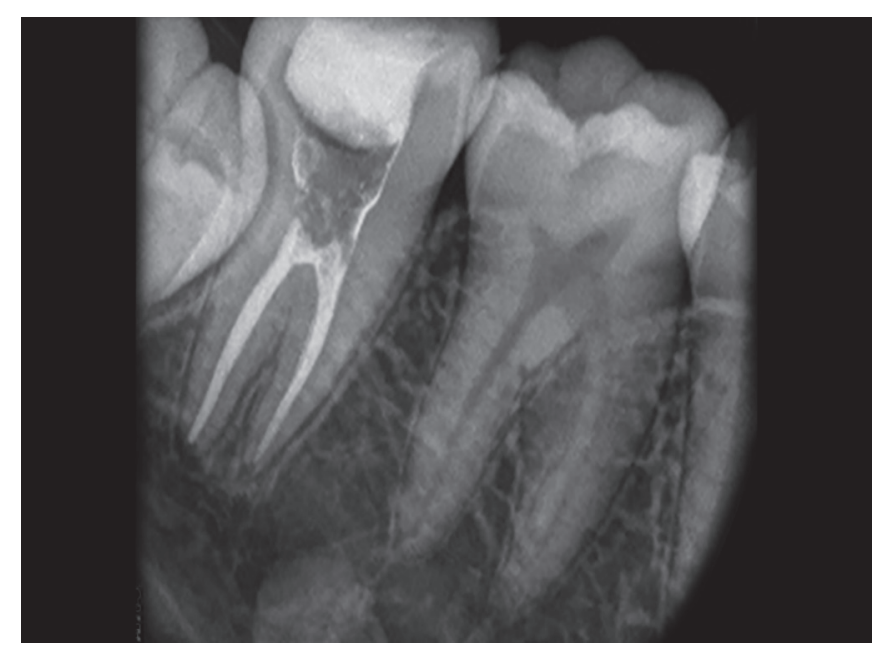

Fig. 6: Postobturation radiograph

or trifurcation of the root to the cementoenamel junction should be greater than the occlusocervical height for a taurodontic tooth. ${ }^{9}$

The long rectangular shape of pulp chamber makes it difficult to locate the canal orifices and subsequently for the preparation of the canals and obturation. Hence, the success of endodontic treatment in taurodontic teeth largely depends on the use of dental microscope. The use of magnification enhances the visualization of the pulpal floor by better illumination of the depths of the cavity, enabling easy identification of the root canal orifices. Due to the complexity of the root canals, use of adjuncts to chemomechanical debridement, such as ultrasonic irrigation is helpful in cleaning and disinfecting the root canal system. ${ }^{10}$

Tsesis et al suggested that a combination of the lateral compaction technique and the warm vertical compaction technique can be used to obturate a taurodont tooth. ${ }^{11}$ Sectional sealing of apical portion of the canal and backfill with thermoplasticized gutta-percha ensures a threedimensional obturation of the canals and also prevents apical extrusion of the material. ${ }^{12}$ In the case presented, the canals were obturated using lateral compaction in the apical region with vertical compaction at the level of canal orifices.

Postendodontic restoration in taurodontic tooth is also a critical player in the long-term clinical success. The lack of a cervical constriction would deprive the tooth of the buttressing effect against excessive loading of the crown. ${ }^{13}$ Moreover, the remaining dentin thickness of these roots is less, leading to chances of root fracture, which is very high in such cases. ${ }^{12}$ Hence, it was decided to reinforce the root canal walls using a combination of glass ionomer restoration followed by light cured composite resin. Incremental composite build-up was done to fill the entire chamber without any voids. The patient was reviewed after 1 year and was found to be asymptomatic. 


\section{CONCLUSION}

The case report presents successful endodontic management of a mesotaurodontic mandibular second molar tooth with 1 year review. Use of magnification, copious irrigation and modified obturation techniques are needed for successful treatment outcome of taurodont teeth.

\section{REFERENCES}

1. Jafarzadeh H, Azarpazhooh A, Mayhall JT. Taurodontism: a review of the condition and endodontic treatment challenges. Int Endod J 2008;41(5):375-388.

2. Manjunatha BS, Kovvuru SK. Taurodontism - A review on its etiology, prevalence and clinical considerations. J Clin Exp Dent 2010;2(4):e187-190.

3. Witkop CJ. Clinical aspects of dental anomalies. Int Dent J 1976;26(4):378-390.

4. Parolia A, Khosla M, Kundabala M. Endodontic management of hypo-, meso- and hypertaurodontism: case reports. Aust Endod J 2012 Apr;38(1):36-41.

5. Joseph M. Endodontic treatment in three taurodontic teeth associated with 48, XXXY Klinefilter syndrome: a review and case report. Oral Surg Oral Med Oral Pathol Oral Radiol Endod 2008;105(5):670-677.

6. Shaw JC. Taurodont teeth in South African races. J Anat 1928;62(4):476-498.

7. Shifman A, Chanannel I. Prevalence of taurodontism found in radiographic dental examination of 1,200 young adult Israeli patients. Community Dent Oral Epidemiol 1978;6(4):200-203.

8. Mangion JJ. Two cases of taurodontism in modern human jaws. Br Dent J 1962;3:309-312.

9. Feichtinger C, Rosiwall B. Taurodontism in human sex chromosome aneuploidy. Arch Oral Biol 1977;22(5):327-329.

10. Simsek N, Keles A, Ocak MS. Endodontic treatment of hypertaurodontism with multiple bilateral taurodontism. J Conserv Dent 2013;16(5):477-479.

11. Tsesis I, Shifman A, Kaufman AY. Taurodontism: an endodontic challenge-report of a case. J Endod 2003;29(5): 353-355.

12. Prakash R, Vishnu C, Suma B, Velmurugan N, Kandaswamy D. Endodontic management of taurodontic teeth. Ind J Dent Res 2005;16(4):177-181.

13. Durr DP, Campos CA, Ayers CS. Clinical significance of Taurodontism. J Am Dent Assoc 1980;100(3):378-381. 